\title{
Pathologic assessment of tumor-associated macrophages and their histologic localization in invasive breast carcinoma
}

\author{
Shorouk E. Mwafy and Dina M. El-Guindy*
}

\begin{abstract}
Background: Tumor-associated macrophages (TAMs) are important in regulating cross-talk between tumor cells and tumor microenvironment. TAMs are involved in multiple steps of tumor progression and invasion. This study aimed to compare CD163 expression with the widely used CD68 pan-macrophage marker in invasive breast carcinoma. Furthermore, it focused on assessing the significance of TAMs localization in relation to clinicopathological parameters.

Results: CD68 and CD163 immunohistochemical expressions within TAMs infiltrating both tumor nest (TN) and tumor stroma (TS) were evaluated in 60 specimens with invasive breast carcinoma. High CD68-positive stromal TAMs was significantly related to larger tumor, nodal metastasis and vascular invasion $(p=0.003,0.037,0.032$, respectively), whereas high CD163-positive stromal TAMs was significantly related to larger tumors, nodal metastasis, stage III tumors, vascular invasion, estrogen receptor (ER) negativity, and triple-negative subtype ( $p=$ $0.023,<0.001,0.001,0.022,0.002,0.017$, respectively). On multivariate analysis, high CD68-positive TAMs infiltrating TS was significantly associated with larger tumor and positive nodal metastasis ( $p=0.006$ and 0.016 , respectively), whereas high CD163 TAMs density within TS was significantly associated with positive vascular invasion, nodal metastasis, and molecular subtypes ( $p=0.003,0.001$, and 0.009 , respectively).

Conclusion: TAMs within tumor stroma and tumor nest have different levels of association with poor prognostic parameters. So, it is of great importance to consider the histologic localization of TAMs in addition to the degree of TAMs infiltration.
\end{abstract}

Keywords: Tumor-associated macrophages (TAMs), CD163, CD68, Breast cancer, Histologic localization

\section{Background}

Breast cancer $(\mathrm{BC})$ is the most commonly diagnosed cancer and the main cause of cancer-related deaths in females worldwide [1]. Initial studies investigating mechanisms responsible for $\mathrm{BC}$ metastatic potential and treatment resistance have focused attention on tumor cells themselves. However, the role of the tumor microenvironment (TME) in tumor progression and treatment resistance have been recently identified [2].

Tumors are composed of malignant and non-malignant cells, which constitute the tumor microenvironment [3]. Tumor-associated macrophages (TAMs) are crucial regulators of cancer cells and microenvironment. They are

\footnotetext{
* Correspondence: dina-elguindy@hotmail.com

Pathology Department, Faculty of Medicine, Tanta University, Tanta, Egypt
}

highly plastic cells that are affected by and are reprogrammed by signals found within TME. Chemotactic factors attract TAMs to tumors which are a rich source of cytokines and proteases for the promotion of invasion, tumor angiogenesis, immune evasion, and suppression of apoptosis [4]

TAMs have a potential ability to differentiate into either M1- or M2-polarized macrophages, which have opposing effects on tumor progression. Classically, activated M1 macrophages release pro-inflammatory cytokines and activate type $1 \mathrm{~T}$ cell response that has a cytotoxic effect on tumor cells, whereas M2 macrophages produce proteolytic enzymes, suppress immune response, and contribute to hypoxia-induced angiogenesis, thus promoting tumor cell proliferation and migration [5]. 
CD68 and CD163 are glycoproteins that are expressed in human monocytes and tissue macrophages. CD68 is a pan-macrophage marker that recognizes both M1 and M2 macrophages, while CD163 is a highly specific monocyte/macrophage marker for polarized M2 macrophages [6, 7].

Previous studies demonstrated that TAMs infiltration is associated with poor clinical outcomes in breast cancer. Also, high level of TAMs infiltration was associated with negative hormone receptor status [8]. TAMs have been considered as a potential target for adjuvant therapy [5].

This study aimed to evaluate CD163 expression compared to the widely used CD68 pan-macrophage marker in invasive breast carcinoma. In addition, it focused on assessing the significance of TAMs localization in relation to clinicopathologic parameters.

\section{Methods}

This retrospective study included 60 primary BC specimens. Formalin-fixed paraffin-embedded blocks were retrieved from the Pathology Department during the period from January 2018 to June 2019. Approval of the institutional ethics committee was obtained.

Eligibility in this study included patients who had pathologically confirmed invasive breast carcinoma of no special type, proper histologic specimens with sufficient tumor tissue, and complete clinicopathologic data. Patients who received chemotherapy or radiotherapy prior to surgery were excluded.

Clinicopathologic data of these patients were obtained from their medical reports. All included cases were classified as invasive carcinoma of no special type according to the World Health Organization (WHO) criteria [9]. Nottingham grading system was used to determine tumor grade $[9,10]$. TNM staging was assessed according to the American Joint Committee on Cancer [11]. Molecular subtypes were categorized as luminal A, luminal B, HER2+, and triple-negative subtype according to the modern molecular classification [12].

\section{Immunohistochemical staining}

Sections (5 um thick) were prepared on positively charged slides and then left to dry for $30 \mathrm{~min}$ at $37^{\circ} \mathrm{C}$. Dako PT Link unit was applied for deparaffinization and antigen retrieval. Both high and low pH EnVisionTM FLEX Target Retrieval Solutions were used reaching 97 ${ }^{\circ} \mathrm{C}$ for $20 \mathrm{~min}$. Immunostaining was carried out with Dako Autostainer Link 48. Antibodies included in this study were CD68 mouse monoclonal antibody (M0876, Dako, Glostrup, Denmark) and CD163 rabbit monoclonal antibody (clone EP324, Medaysis, CA, USA). In brief, slides were left in Peroxidase-Blocking Reagent for 5 min, incubated with primary antibodies for 20-30 min, horseradish peroxidase (HRP) polymer reagent for 20 min, and diaminobenzidine (DAB) chromogen/substrate working solution for $10 \mathrm{~min}$. Finally, counterstaining with hematoxylin was done.

\section{Quantification of TAMs}

All CD68 and CD163 stained slides were examined to determine areas with the highest levels of tumorassociated macrophages (TAMs) infiltration. For each case, three hotspots in a high-power field $(\times 400)$ were decided for counting TAMs. TAMs were counted manually using the plug-in "cell counter" in the ImageJ software. TAMs were counted in both tumor nest (TN) and tumor stroma (TS). TAMs within TN represent macrophages within tumor cell nests and indirect contact with tumor cells, whereas stromal TAMs were defined as macrophages infiltrating tumor stroma of the invasive carcinoma. Cases were then assigned into low and high groups considering the median density of TAMs infiltration in both TN and TS as a cut-off point [13].

Cases were further grouped according to CD68- and CD163-positive TAMs density in both TN and TS as follows: $\mathrm{TN}^{\mathrm{Low}}$ and $\mathrm{TS}^{\mathrm{Low}}$ included cases with low TAMs density in both TN and TS, $\mathrm{TN}^{\mathrm{High}}$ and $\mathrm{TS}^{\mathrm{Low}}$ included cases with high TAMs density in TN and low TAMs density in TS, $\mathrm{TN}^{\mathrm{High}}$ and $\mathrm{TS}^{\mathrm{High}}$ included cases with high TAMs density in both TN and TS, $\mathrm{TN}^{\mathrm{Low}}$ and $\mathrm{TS}^{\text {High }}$ included cases with low TAMs density in TN and high TAMs density in TS.

\section{Statistical analysis}

Statistical analysis was performed using Statistical Package for Social Science (SPSS version 23.0). Data were presented as mean \pm SD for numerical variables and frequencies for categorical ones. Analyzing relations between TAMs infiltration and clinicopathologic variables was carried out using chi-square $\left(\chi^{2}\right)$. Fisher exact and Monte-Carlo tests were used when appropriate. Column proportion test was used for pairwise comparisons when the omnibus test was significant. The $p$ values were adjusted with Bonferroni method. Normality of numerical variables was determined using Shapiro-Wilk test. Independent student $t$ test and analysis of variance (ANOVA) test were used to compare means of numerical variables. A multivariate logistic regression analysis was performed to identify the variables that were independently associated with TAMs infiltration. $p$ values $<$ 0.05 were considered statistically significant.

\section{Results}

Clinicopathologic characteristics

Clinicopathologic characteristics of the studied cases are illustrated in Table 1. Mean age of the studied BC cases was $51.22+11.64$ years. In $39(65 \%)$ cases, tumor 
Table 1 Clinicopathological characteristics of the studied cases

\begin{tabular}{|c|c|}
\hline Variable & Total \\
\hline Age (years) mean \pm SD & $51.22 \pm 11.64$ \\
\hline \multicolumn{2}{|l|}{ Size } \\
\hline$<5 \mathrm{~cm}$ & $39(65)$ \\
\hline$\geq 5 \mathrm{~cm}$ & $21(35)$ \\
\hline \multicolumn{2}{|l|}{ Grade } \\
\hline Gll & $32(53.3)$ \\
\hline GIII & $28(46.7)$ \\
\hline \multicolumn{2}{|l|}{ Nodal metastasis } \\
\hline Negative & $26(43.3)$ \\
\hline Positive & $34(56.7)$ \\
\hline \multicolumn{2}{|l|}{ Staging } \\
\hline । & $3(5)$ \\
\hline$\|$ & $32(53.3)$ \\
\hline III & $25(41.7)$ \\
\hline \multicolumn{2}{|l|}{ Vascular invasion } \\
\hline Negative & $22(36.7)$ \\
\hline Positive & $38(63.3)$ \\
\hline \multicolumn{2}{|l|}{ ER } \\
\hline Negative & $13(21.7)$ \\
\hline Positive & $47(78.3)$ \\
\hline \multicolumn{2}{|l|}{$P R$} \\
\hline Negative & $26(43.3)$ \\
\hline Positive & $34(56.7)$ \\
\hline \multicolumn{2}{|l|}{ Her2 } \\
\hline Negative & $45(75)$ \\
\hline Positive & $15(25)$ \\
\hline \multicolumn{2}{|l|}{ Ki-67 } \\
\hline$\leq 14 \%$ & $29(48.3)$ \\
\hline$>14 \%$ & $31(51.7)$ \\
\hline \multicolumn{2}{|l|}{ Molecular subtypes } \\
\hline Luminal A & $27(45)$ \\
\hline Luminal B & $24(40)$ \\
\hline Triple negative & $9(15)$ \\
\hline
\end{tabular}

$E R$ estrogen receptor, $P R$ progesterone receptor

measured $<5 \mathrm{~cm}$ in its greatest dimension. GII tumors constituted $32(53.3 \%)$ cases whereas $28(46.7 \%)$ cases were poorly differentiated. Concerning tumor stage, 25 (41.7\%) cases were stage III, $32(53.3 \%)$ cases were stage II, while only $3(5 \%)$ cases were stage I. Positive nodal metastasis was detected in $34(56.7 \%)$ cases, whereas positive vascular invasion was identified in 38 (63.3\%) cases.

As regards hormone receptor status, 47 (78.3\%) cases were estrogen receptor (ER)-positive and 34 (56.7\%) positively expressed progesterone receptor (PR). Forty- five $(75 \%)$ cases were negative for Her2 expression. Ki67 proliferation index was $>14 \%$ in $31(51.7 \%)$ cases and $\leq 14 \%$ in the remaining $29(48.3 \%)$ cases. Referring to the molecular subtypes, 27 (45\%) cases were luminal A, $24(40 \%)$ cases were luminal B while the remaining 9 (15\%) cases were triple-negative tumors.

\section{Relation between CD68 expression in both tumor nest and stroma and clinicopathologic characteristics}

Significant relations were detected between CD68-positive TAMs infiltration within TS and tumor size, nodal metastasis and vascular invasion. CD68-positive TAMs infiltrating TS was significantly higher in $16(76.2 \%)$ tumors measuring $>5 \mathrm{~cm}$ in its greatest dimension $(p=0.003), 21$ $(61.8 \%)$ cases positive for nodal metastasis $(p=0.037)$ and $23(60.5 \%)$ tumors with positive vascular invasion $(p=$ 0.032). On the contrary, CD68-positive TAMs in TN was not significantly associated with any clinicopathological features. On multivariate logistic regression analysis, high CD68-positive stromal TAMs was significantly associated with larger tumor $(\mathrm{OR}=0.044 ; 95 \% \mathrm{CI}=0.005-0.413 ; p=$ $0.006)$ and positive nodal metastasis $(\mathrm{OR}=0.074 ; 95 \% \mathrm{CI}$ $=0.009-0.621 ; p=0.016$ ) (Tables 2 and 3, Fig. 1 ).

\section{Relation between combined densities of CD68-positive TAMs in both tumor nest and stroma and clinicopathologic characteristics}

Tumors with a combined high density of CD68-positive TAMs in both TN and TS were significantly associated with larger tumors $(p=0.001)$. In pairwise comparison, CD68-positive $\mathrm{TN}^{\text {High }}$ and $\mathrm{TS}^{\text {High }}$ cases showed the highest proportion of tumors measuring $\geq 5 \mathrm{~cm}$ in their greatest dimension (57.2\%) compared to cases with CD68-positive $\mathrm{TN}^{\mathrm{Low}}$ and $\mathrm{TS}^{\mathrm{Low}}(19 \%), \mathrm{TN}^{\mathrm{High}}$ and $\mathrm{TS}^{\mathrm{Low}}(4.8 \%)$, and $\mathrm{TN}^{\mathrm{Low}}$ and $\mathrm{TS}^{\mathrm{High}}$ (19\%) (pairwise $p=$ $0.003,<0.001,0.026$, respectively). As regards vascular invasion, CD68-positive $\mathrm{TN}^{\mathrm{High}}$ and $\mathrm{TS}^{\mathrm{High}}$ cases were significantly higher in cases with positive vascular invasion compared to CD68-positive $\mathrm{TN}^{\mathrm{High}}$ and $\mathrm{TS}^{\mathrm{Low}}$ ones (pairwise $p=0.048$ ). These relations are demonstrated in Table 4.

\section{Relation between CD163 expression in both tumor nest and stroma and clinicopathologic characteristics}

There were statistically significant associations between CD163-positive TAMs infiltrating TS and tumor size, lymph node (LN) metastasis, tumor stage, and vascular invasion. High density of CD163-positive TAMs infiltrating TS was detected in 14 (66.7\%) tumors measuring $\geq 5$ $\mathrm{cm}$ in their greatest dimension $(p=0.023), 23(67.6 \%)$ cases with positive LN metastasis $(p<0.001), 18(72 \%)$ stage III cases $(p=0.001)$, and in $22(57.9 \%)$ cases in which vascular invasion was identified $(p=0.022)$. Moreover, 11 (84.6\%) ER-negative cases displayed high 
Table 2 Relation between CD68 expression in both tumor nest and stroma and clinicopathologic characteristics

\begin{tabular}{|c|c|c|c|c|c|c|c|}
\hline \multirow[t]{2}{*}{ Variable } & \multirow[t]{2}{*}{ Total } & \multicolumn{3}{|c|}{ CD68 in tumor nest } & \multicolumn{3}{|c|}{ CD68 in tumor stroma } \\
\hline & & $\begin{array}{l}\text { Low } \\
N=31 \\
N(\%)\end{array}$ & $\begin{array}{l}\text { High } \\
N=29 \\
N(\%)\end{array}$ & $p$ & $\begin{array}{l}\text { Low } \\
N=30 \\
N(\%)\end{array}$ & $\begin{array}{l}\text { High } \\
N=30 \\
N(\%)\end{array}$ & $p$ \\
\hline \multicolumn{2}{|c|}{ Age (years) mean \pm SD } & $52.81 \pm 11.88$ & $49.52 \pm 11.34$ & 0.278 & $52.17 \pm 12.19$ & $50.27 \pm 11.19$ & 0.532 \\
\hline \multicolumn{8}{|l|}{ Size } \\
\hline$<5 \mathrm{~cm}$ & 39 & $23(59)$ & $16(41)$ & \multirow[t]{2}{*}{0.123} & $25(64.1)$ & $14(35.9)$ & \multirow[t]{2}{*}{$0.003^{*}$} \\
\hline$\geq 5 \mathrm{~cm}$ & 21 & $8(38.1)$ & $13(61.9)$ & & $5(23.8)$ & $16(76.2)$ & \\
\hline \multicolumn{8}{|l|}{ Grade } \\
\hline Gll & 32 & $18(56.3)$ & $14(43.8)$ & \multirow[t]{2}{*}{0.448} & $18(56.3)$ & $14(43.8)$ & \multirow[t]{2}{*}{0.301} \\
\hline GIII & 28 & $13(46.4)$ & 15 (53.6) & & $12(42.9)$ & $16(57.1)$ & \\
\hline \multicolumn{8}{|l|}{ Nodal metastasis } \\
\hline Negative & 26 & $17(65.4)$ & $9(34.6)$ & \multirow[t]{2}{*}{0.063} & $17(65.4)$ & $9(34.6)$ & \multirow[t]{2}{*}{$0.037^{*}$} \\
\hline Positive & 34 & $14(41.2)$ & $20(58.8)$ & & $13(38.2)$ & $21(61.8)$ & \\
\hline \multicolumn{8}{|l|}{ Staging } \\
\hline I & 3 & $2(66.7)$ & $1(33.3)$ & \multirow[t]{3}{*}{0.118} & $3(100)$ & $0(0)$ & \multirow[t]{3}{*}{0.153} \\
\hline$\|$ & 32 & $20(62.5)$ & $12(37.5)$ & & $17(53.1)$ & $15(46.9)$ & \\
\hline III & 25 & $9(36)$ & $16(64)$ & & $10(40)$ & $15(60)$ & \\
\hline \multicolumn{8}{|l|}{ Vascular invasion } \\
\hline Negative & 22 & $9(40.9)$ & $13(59.1)$ & \multirow[t]{2}{*}{0.205} & $15(68.2)$ & $7(31.8)$ & \multirow[t]{2}{*}{$0.032^{*}$} \\
\hline Positive & 38 & $22(57.9)$ & $16(42.1)$ & & 15 (39.5) & $23(60.5)$ & \\
\hline \multicolumn{8}{|l|}{ ER } \\
\hline Negative & 13 & $6(46.2)$ & $7(53.8)$ & \multirow[t]{2}{*}{0.653} & $7(53.8)$ & $6(46.2)$ & \multirow[t]{2}{*}{0.754} \\
\hline Positive & 47 & $25(53.2)$ & $22(46.8)$ & & $23(48.9)$ & $24(51.1)$ & \\
\hline \multicolumn{8}{|l|}{ PR } \\
\hline Negative & 26 & $14(53.8)$ & $12(46.2)$ & \multirow[t]{2}{*}{0.768} & $14(53.8)$ & $12(46.2)$ & \multirow[t]{2}{*}{0.602} \\
\hline Positive & 34 & $17(50)$ & $17(50)$ & & $16(47.1)$ & $18(52.9)$ & \\
\hline \multicolumn{8}{|l|}{ Her2 } \\
\hline Negative & 45 & $22(48.9)$ & $23(51.1)$ & \multirow[t]{2}{*}{0.456} & $23(51.1)$ & $22(48.9)$ & 0.766 \\
\hline Positive & 15 & $9(60)$ & $6(40)$ & & $7(46.7)$ & $8(53.3)$ & \\
\hline Ki-67 & & & & & & & \\
\hline$\leq 14 \%$ & 29 & $16(55.2)$ & $13(44.8)$ & 0.599 & $15(51.7)$ & $14(48.3)$ & 0.796 \\
\hline$>14 \%$ & 31 & $15(48.4)$ & $16(51.6)$ & & $15(48.4)$ & 16 (51.6) & \\
\hline Molecular subtype & & & & & & & \\
\hline Luminal A & 27 & $15(55.6)$ & $12(44.4)$ & 0.535 & $14(51.9)$ & $13(48.1)$ & 0.475 \\
\hline Luminal B & 24 & $13(54.2)$ & $11(45.8)$ & & $10(41.7)$ & $14(58.3)$ & \\
\hline Triple negative & 9 & $3(33.3)$ & $6(66.7)$ & & $6(66.7)$ & $3(33.3)$ & \\
\hline
\end{tabular}

$E R$ estrogen receptor, $P R$ progesterone receptors

*Statistically significant

Table 3 Multivariate analysis of clinicopathological data and CD68 positive TAMs within tumor stroma

\begin{tabular}{lll}
\hline Variables & OR $(95 \% \mathrm{Cl})$ & $p$ \\
\hline Size & $0.044(0.005-0.413)$ & $0.006^{*}$ \\
Nodal metastasis & $0.074(0.009-0.621)$ & $0.016^{*}$ \\
Vascular invasion & $1.364(0.241-7.734)$ & 0.726 \\
\hline
\end{tabular}

Cl confidence interval, OR odds ratio, TAMs tumor-associated macrophage *Statistically significant density of CD163-positive TAMs in TS $(p=0.002)$. As regards molecular subtypes, CD163-positive TAMs in TS was significantly high in triple-negative cases and low in luminal A cases $(p=0.017)$. Dealing with CD163positive TAMs within $\mathrm{TN}$, high density was detected in $19(67.9 \%)$ grade III tumors $(p=0.005)$, and in 21 (67.7\%) tumors with high ki-67 proliferation index $(p=$ 0.002). Also, a significant association was observed 


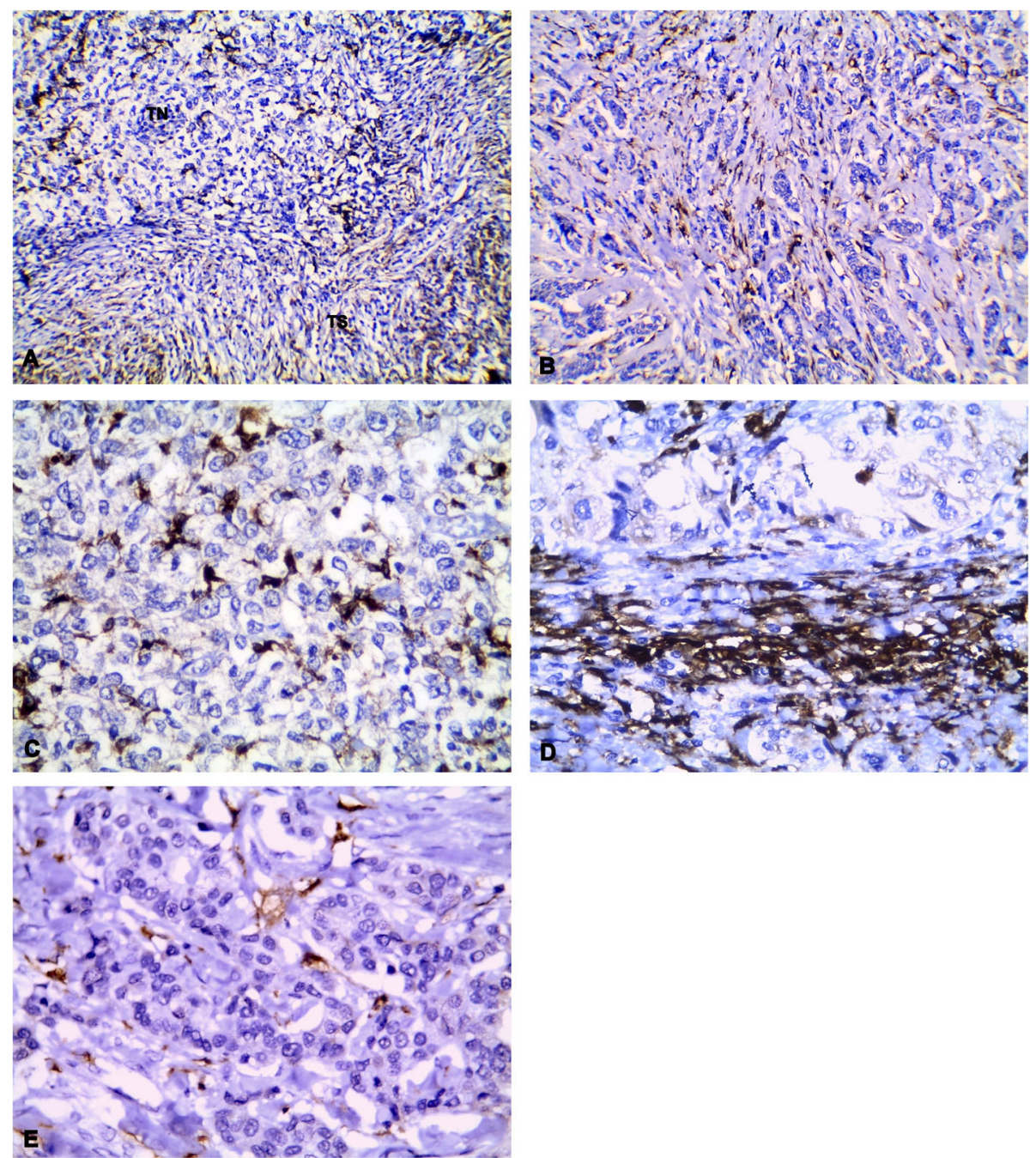

Fig. 1 CD68-positive tumor-associated macrophages (TAMs). a CD68-positive TAMs mainly in tumor stroma (TS) with few within tumor nest (TN) in GIII case (× 200). b CD68-positive TAMs within tumor stroma in GIl case (× 200). c High density of CD68-positive TAMs within tumor nest in GIII case $(\times 400)$. $\mathbf{d}$ High density of CD68-positive TAMs infiltrating tumor stroma in Glll case (× 400). e Low density of CD68-positive TAMs in both

tumor nest and stroma in Gll case $(\times 400)$

between low density of CD163-positive TAMs within TN and luminal A subtype $(p=0.001)$. On multivariate logistic regression analysis, high CD163 TAMs density within TS was significantly associated with positive vascular invasion $(\mathrm{OR}=0.026 ; 95 \% \mathrm{CI}=0.002-0.284 ; p=$ $0.003)$, nodal metastasis $(\mathrm{OR}=0.068 ; 95 \% \mathrm{CI}=0.014-$ $0.331 ; p=0.001)$, and molecular subtypes $(p=0.009)$, whereas no significant associations were detected between CD163-positive TAMs within TN and clinicopathologic characteristics (Tables 5 and 6, Fig. 2).

\section{Relation between combined densities of CD163-positive TAMs in both tumor nest and stroma and clinicopathologic characteristics}

CD163-positive $\mathrm{TN}^{\mathrm{High}}$ and $\mathrm{TS}^{\text {High }}$ cases were significantly associated with GIII tumors, positive nodal metastasis, stage III tumors, ER negativity, high Ki-67 proliferation index compared to CD163-positive $\mathrm{TN}^{\mathrm{Low}}$ and $\mathrm{TS}^{\mathrm{Low}}$ (pairwise $p=0.029,0.003,0.018,0.011,0.012$, respectively) denoting that high density of CD163-positive is associated with poor prognostic parameters. CD163-positive $\mathrm{TN}^{\text {High }}$ and $\mathrm{TS}^{\mathrm{High}}$ cases were associated with higher GIII tumors compared to CD163-positive $\mathrm{TN}^{\mathrm{Low}}$ and $\mathrm{TS}^{\mathrm{High}}$ (pairwise $p=0.007)$. Also, CD163-positive $\mathrm{TN}^{\mathrm{Low}}$ and $\mathrm{TS}^{\mathrm{High}}$ cases were significantly related to positive nodal metastasis and stage III tumors compared to CD163-positive $\mathrm{TN}^{\mathrm{Low}}$ and TS $^{\text {Low }}$ (pairwise $p=0.011$ and 0.018 , respectively). CD163-positive $\mathrm{TN}^{\mathrm{High}}$ and $\mathrm{TS}^{\mathrm{Low}}$ cases were significantly associated with high Ki-67 compared to CD163-positive $\mathrm{TN}^{\mathrm{Low}}$ and $\mathrm{TS}^{\mathrm{Low}}$ (pairwise $p=0.012$ ). Regarding molecular subtypes, CD163-positive $\mathrm{TN}^{\mathrm{Low}}$ and $\mathrm{TS}^{\mathrm{Low}}$ had the highest proportion of luminal A tumors (48.2\%) compared 
Table 4 Relation between combined densities of CD68 expression in both tumor nest and stroma and clinicopathologic characteristics

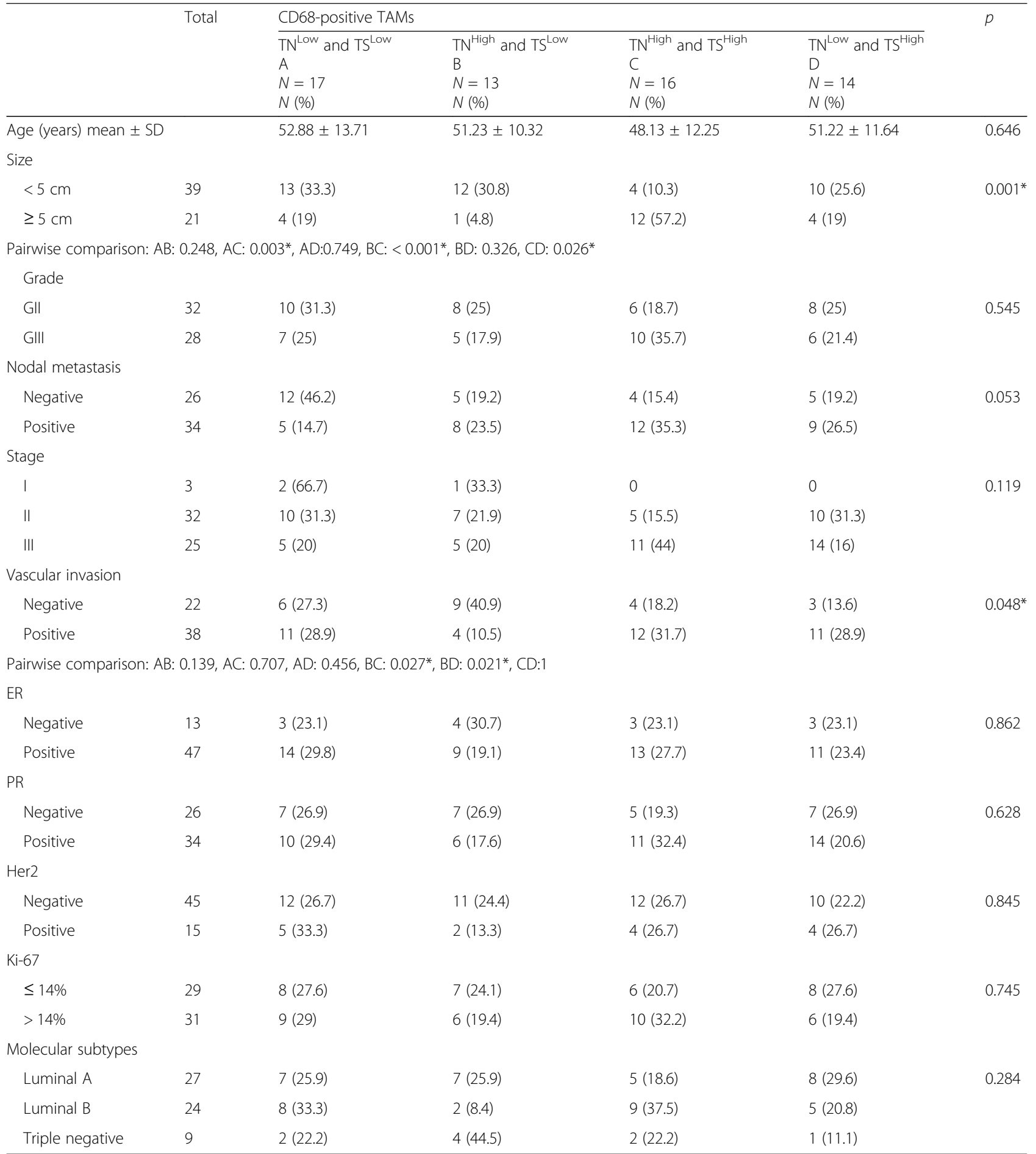

$E R$ estrogen receptor, $P R$ progesterone receptor, TAMs tumor-associated macrophage, $T N$ tumor nest, $T S$ tumor stroma *Statistically significant

to CD163-positive $\mathrm{TN}^{\text {High }}$ and $\mathrm{TS}^{\text {Low }}(7.4 \%)$ and $\mathrm{TN}^{\mathrm{High}}$ and $\mathrm{TS}^{\mathrm{High}}$ cases (14.8\%) (pairwise $p=0.002$ and 0.011 , respectively), whereas CD163-positive $\mathrm{TN}^{\mathrm{Low}}$ and $\mathrm{TS}^{\text {High }}$ cases had the highest proportion of triple-negative cases (44.4\%) compared to CD163-positive $\mathrm{TN}^{\text {High }}$ and $\mathrm{TS}^{\mathrm{Low}}$ (11.2\%) (pairwise $p=0.002$ ) as shown in Table 7. 
Table 5 Relation between CD163 expression in both tumor nest and stroma and clinicopathologic characteristics

\begin{tabular}{|c|c|c|c|c|c|c|c|}
\hline \multirow[t]{2}{*}{ Variable } & \multirow[t]{2}{*}{ Total } & \multicolumn{3}{|c|}{ CD163 in tumor nest } & \multicolumn{3}{|c|}{ CD163 in tumor stroma } \\
\hline & & $\begin{array}{l}\text { Low } \\
N=31 \\
N(\%)\end{array}$ & $\begin{array}{l}\text { High } \\
N=29 \\
N(\%)\end{array}$ & $p$ & $\begin{array}{l}\text { Low } \\
N=32 \\
N(\%)\end{array}$ & $\begin{array}{l}\text { High } \\
N=28 \\
N(\%)\end{array}$ & $p$ \\
\hline \multicolumn{2}{|c|}{ Age (years) mean \pm SD } & $52.68 \pm 12.99$ & $49.66 \pm 9.99$ & 0.319 & $49.41 \pm 11.92$ & $53.29 \pm 11.15$ & 0.200 \\
\hline \multicolumn{8}{|l|}{ Size } \\
\hline$<5 \mathrm{~cm}$ & 39 & $21(53.8)$ & $18(46.2)$ & \multirow[t]{2}{*}{0.645} & $25(64.1)$ & $14(35.9)$ & \multirow[t]{2}{*}{$0.023^{*}$} \\
\hline$\geq 5 \mathrm{~cm}$ & 21 & $10(47.6)$ & $11(52.4)$ & & $7(33.3)$ & $14(66.7)$ & \\
\hline \multicolumn{8}{|l|}{ Grade } \\
\hline Gll & 32 & $22(68.8)$ & $10(31.3)$ & \multirow[t]{2}{*}{$0.005^{*}$} & $18(56.3)$ & $14(43.7)$ & \multirow[t]{2}{*}{0.628} \\
\hline GIII & 28 & $9(32.1)$ & $19(67.9)$ & & $14(50)$ & $14(50)$ & \\
\hline \multicolumn{8}{|l|}{ Nodal metastasis } \\
\hline Negative & 26 & $15(57.7)$ & $11(42.3)$ & \multirow[t]{2}{*}{0.414} & $21(80.8)$ & $5(19.2)$ & \multirow[t]{2}{*}{$<0.001^{*}$} \\
\hline Positive & 34 & $16(47.1)$ & $18(52.9)$ & & $11(32.4)$ & $23(67.6)$ & \\
\hline \multicolumn{8}{|l|}{ Staging } \\
\hline I & 3 & $3(100)$ & $0(0)$ & \multirow[t]{3}{*}{0.324} & $3(100)$ & $0(0)$ & \multirow[t]{3}{*}{$0.001^{*}$} \\
\hline$\|$ & 32 & $16(50)$ & $16(50)$ & & $22(68.8)$ & $10(31.2)$ & \\
\hline III & 25 & $12(48)$ & $13(52)$ & & $7(28)$ & $18(72)$ & \\
\hline \multicolumn{8}{|l|}{ Vascular invasion } \\
\hline Negative & 22 & $13(59.1)$ & $9(40.9)$ & \multirow[t]{2}{*}{0.381} & 16 (72.7) & $6(27.3)$ & \multirow[t]{2}{*}{$0.022^{*}$} \\
\hline Positive & 38 & $18(47.4)$ & $20(52.6)$ & & $16(42.1)$ & $22(57.9)$ & \\
\hline \multicolumn{8}{|l|}{ ER } \\
\hline Negative & 13 & $5(38.5)$ & $8(61.5)$ & \multirow[t]{2}{*}{0.282} & $2(15.4)$ & $11(84.6)$ & \multirow[t]{2}{*}{$0.002^{*}$} \\
\hline Positive & 47 & $26(55.3)$ & $21(44.7)$ & & $30(63.8)$ & 17 (36.2) & \\
\hline \multicolumn{8}{|l|}{$P R$} \\
\hline Negative & 26 & $13(50)$ & $13(50)$ & \multirow[t]{2}{*}{0.821} & $12(46.2)$ & $14(53.8)$ & \multirow[t]{2}{*}{0.330} \\
\hline Positive & 34 & $18(52.9)$ & $16(47.1)$ & & $20(58.8)$ & $14(41.2)$ & \\
\hline \multicolumn{8}{|l|}{ Her2 } \\
\hline Negative & 41 & $25(55.6)$ & $20(44.4)$ & \multirow[t]{2}{*}{0.296} & $21(46.7)$ & $24(53.3)$ & 0.084 \\
\hline Positive & 19 & $6(40)$ & $9(60)$ & & $11(73.3)$ & $4(26.7)$ & \\
\hline Ki-67 & & & & & & & \\
\hline$\leq 14 \%$ & 29 & $21(72.4)$ & $8(27.6)$ & $0.002^{*}$ & 17 (58.6) & $12(41.4)$ & 0.427 \\
\hline$>14 \%$ & 31 & $10(32.3)$ & $21(67.7)$ & & $15(48.4)$ & 16 (51.6) & \\
\hline Molecular subtype & & & & & & & \\
\hline Luminal A & 27 & $21(77.8)$ & $6(22.2)$ & $0.001^{*}$ & 15 (55.6) & $12(44.4)$ & $0.017^{*}$ \\
\hline Luminal B & 24 & $6(25)$ & $18(75)$ & & $16(66.7)$ & $8(33.3)$ & \\
\hline Triple negative & 9 & $4(44.4)$ & $4(55.6)$ & & $1(11.1)$ & $8(88.9)$ & \\
\hline
\end{tabular}

ER estrogen receptor, PR progesterone receptor

*Statistically significant

\section{Discussion}

Breast cancer is the most frequent malignancy among females worldwide. Traditional therapies against breast cancer have been designed to attack tumor cells themselves. Recently, studies have focused on targeting tumor microenvironment in order to reduce treatment resistance and improve patients outcomes [14].
Tumor-associated macrophages are crucial regulators of cancer cells and microenvironment. They modulate tumorigenesis and adjust the response to therapy. Several studies have reported that TAMs are related to poor prognosis in different tumors as hepatocellular carcinoma, gastric cancer, and lung cancer [15]. Several markers are used to label macrophages. CD68 identifies 
Table 6 Multivariate analysis of clinicopathological data and CD163 positive TAMs within tumor stroma

\begin{tabular}{|c|c|c|}
\hline Variables & OR (95\% Cl) & $p$ \\
\hline \multicolumn{3}{|l|}{ Step 1} \\
\hline Nodal metastasis & $0.114(0.034-0.382)$ & $<0.001^{*}$ \\
\hline \multicolumn{3}{|l|}{ Step 2} \\
\hline Nodal metastasis & $0.121(0.033-0.448)$ & $0.002^{*}$ \\
\hline ER & $8.859(1.492-52.287)$ & $0.016^{*}$ \\
\hline \multicolumn{3}{|l|}{ Step 3} \\
\hline Vascular invasion & $0.085(0.014-0.495)$ & $0.006^{*}$ \\
\hline Nodal metastasis & $0.091(0.021-0.389)$ & $0.001^{*}$ \\
\hline ER & $25.52(2.528-257.5)$ & $0.006^{*}$ \\
\hline \multicolumn{3}{|l|}{ Step 4} \\
\hline Vascular invasion & $0.028(0.003-0.315)$ & $0.004^{*}$ \\
\hline Nodal metastasis & $0.071(0.015-0.348)$ & $0.001^{*}$ \\
\hline Molecular subtypes & & 0.082 \\
\hline Luminal A & $0.026(0.001-2.381)$ & 0.113 \\
\hline Luminal B & $0.009(0.001-0.818)$ & $0.041^{*}$ \\
\hline Triple negative & Reference & \\
\hline ER & $2.966(0.147-59.96)$ & 0.478 \\
\hline \multicolumn{3}{|l|}{ Step 5} \\
\hline Vascular invasion & $0.026(0.002-0.284)$ & $0.003^{*}$ \\
\hline Nodal metastasis & $0.068(0.014-0.331)$ & $0.001^{*}$ \\
\hline Molecular subtypes & & $0.009^{*}$ \\
\hline Luminal A & $0.008(0.001-0.231)$ & $0.005^{*}$ \\
\hline Luminal B & $0.003(0.001-0.124)$ & $0.002^{*}$ \\
\hline Triple negative & Reference & \\
\hline
\end{tabular}

$C l$ confidence interval, ER estrogen receptor, OR odds ratio, TAMs tumor-associated macrophage

*Statistically significant

both tumoricidal M1 and tumor-promoting M2 macrophages, whereas CD163 is expressed principally by M2 macrophages [16].

Studies investigated TAMs infiltration within BC were remarkably variable. They used different markers and methods to assess macrophages. Most of them used CD68 alone to assess macrophages $[13,17]$, while others combined both CD68 and CD163 [18, 19]. Some studies assessed TAMs in different localization (stroma and nest) $[13,17]$, while others neglected the location of TAMs and counted total TAMs within the tumor [20,21].

This study aimed to evaluate CD163 expression compared to the widely used CD68 pan-macrophage marker in breast invasive ductal carcinoma. In addition, it focused on assessing the significance of TAMs localization in relation to clinicopathologic parameters.

As regards TAMs infiltration within TN, the present work revealed that high density of CD163-positive TAMs within TN was significantly associated with high tumor grade and increased Ki-67 proliferation index. On the other hand, there was lack of significant associations between high density of CD68-positive TAMs infiltration and all included clinicopathologic parameters.

Gwak et al. and Jeong et al. reported that a high density of TAMs was related to high tumor grade and higher Ki-67 expression in both locations (tumor nest and tumor stroma) [13, 18]. Similarly, studies by Ni et al. and Sousa et al. revealed significant relations between high infiltration of both CD68-positive and CD163positive TAMs, without addressing specific location, and high histologic grade and increased Ki-67 proliferation index $[20,21]$.

On the contrary, Ch'ng et al. and Yang and his colleagues demonstrated that increased TAMs in the stroma, not within tumor nest, were correlated with higher tumor grade $[17,22]$. However, Yuan et al. noticed a lack of significant association between CD68-positive TAMs density and tumor grade [23].

It was proposed that high-grade tumors may elaborate higher levels of cytokines that recruit and modulate macrophages as monocyte colony-stimulating factors, interleukin-10, and/or transforming growth factor- $\beta$ resulting in increased density of CD163-positive TAMs within high-grade tumors [21]. Moreover, TAMs may secrete different cytokines and growth factors that provide mitogenic signals to malignant cells [17].

As regards TAMs infiltration within tumor stroma, the present study reported significant associations between stromal TAMs infiltration and poor prognostic parameters. Infiltration of tumor stroma with high density of CD68-positive TAMs was significantly related to large tumor size, vascular invasion, and positive nodal metastasis, whereas, high density of stromal CD163-positive TAMs was significantly associated with large tumor size, positive vascular invasion, the presence of nodal metastasis, advanced stage, and ER-negative expression.

This was in accordance with several studies. Ch'ng et al. reported that only elevated stromal CD68-positive TAMs were associated with poor prognostic features [17]. Also, Medrek et al. showed that high density of only CD68- and CD163-positive stromal TAMs were related to larger tumors and inversely correlated with ER-positive expression and luminal A subtype [19]. Moreover, a study by Gwak et al. showed that high density of CD68-positive TAMs, in both tumor nest and stroma, was associated with aggressive histologic features [13].

Dealing with molecular subtypes, this study observed significant associations between CD163-positive TAMs in both TN and TS and molecular subtypes. Most of triple-negative tumors was associated with high density of CD163 positive TAMs. Whereas, luminal A tumors were accompanied by low levels of CD163 positive TAMs within both TN and TS. Similar results were reported by others $[13,18,19]$. A study by Stossi et al. has 

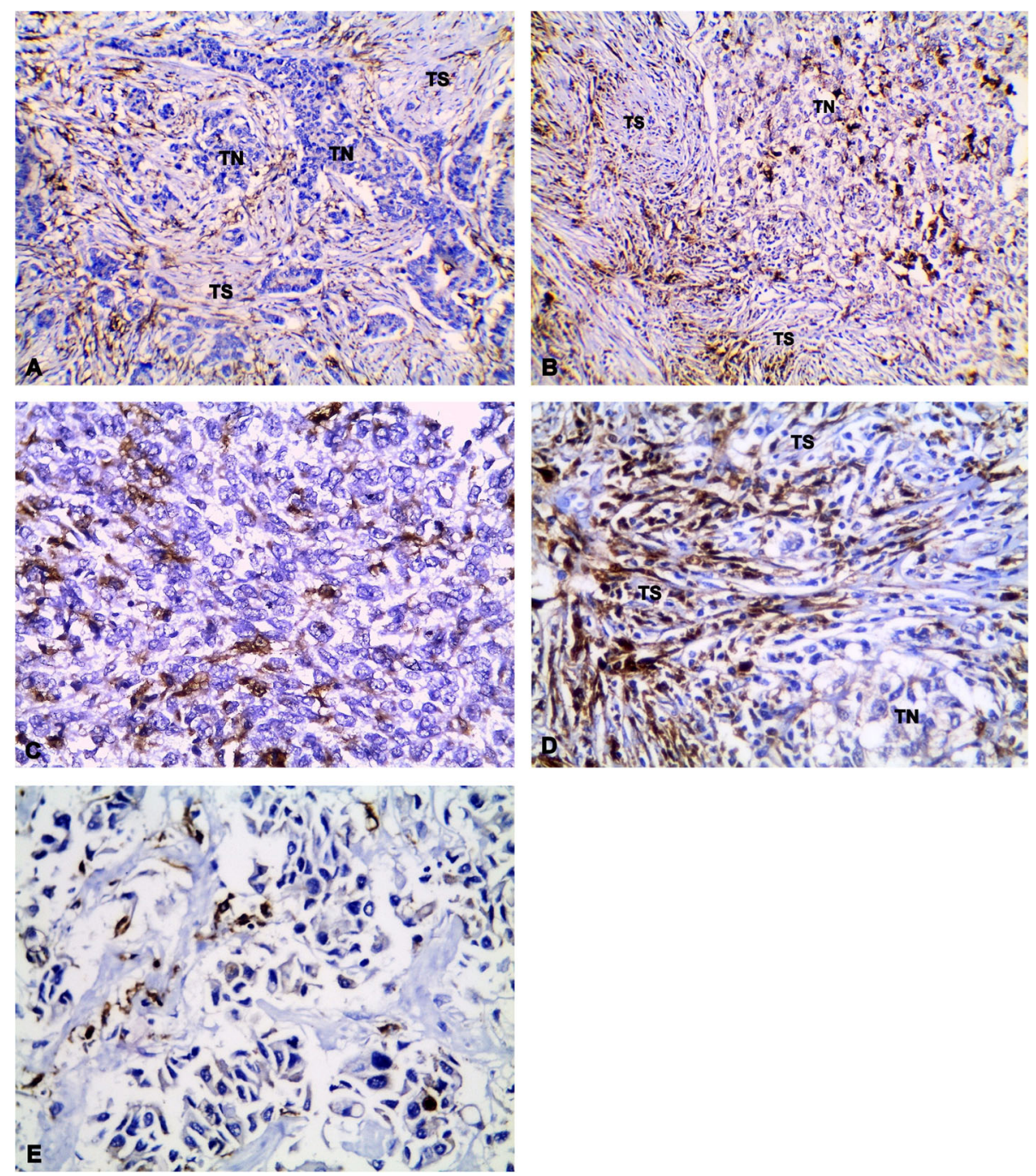
(TN) in GIl case $(\times 200)$. b CD163-positive TAMs within both tumor nest and stroma in Glll case $(\times 200)$. c High density of CD163-positive TAMs infiltrating tumor nest in GIII case $(\times 400)$. d High density of CD163-positive TAMs within tumor stroma in GIII tumor $(\times 400)$. e Low density of CD163-positive TAMs in both tumor nest and stroma in Gll case $(\times 400)$

demonstrated that conditioned media from macrophages was able to stimulate different pathways inside BC cells that were important for the downregulation of ER expression [24].

Some studies evaluated total TAMs without considering TAMs localization and reported that TAMs were associated with an unfavorable prognosis [20, 23, 25]. The present work demonstrated that stromal TAMs were related to more aggressive behavior than that within the tumor nest. It was suggested that TAMs exert its function through regulating immune response within tumor stroma rather than by direct interaction with tumor cells [22].

The role of TAMs in infiltrating tumor nest differs by tumor type. High density of TAMs within tumor nest correlates with better prognosis in endometrial and gastric carcinomas. However, in malignant melanomas and esophageal cancers, TAMs within tumor nest was found to be associated with poor patient outcome [26]. In the tumor stroma, macrophages are recruited by cytokines produced by malignant cells. Most TAMs in the tumor microenvironment are mainly of M2-like phenotype that secretes high levels of cytokines and thus enhances tumor progression. Moreover, TAMs prevent infiltration and action of CD8+ cytotoxic T lymphocytes in attacking tumor cells [2].

In addition, TAMs express vascular endothelial growth factor (VEGF) and activate angiogenesis, and stimulate tumor cell proliferation and metastasis Moreover, TAMs can generate proteases that degrade extracellular matrix and thus enhance tumor cell invasion [27]. 
Table 7 Relation between combined densities of CD163 expression in both tumor nest and stroma and clinicopathologic characteristics

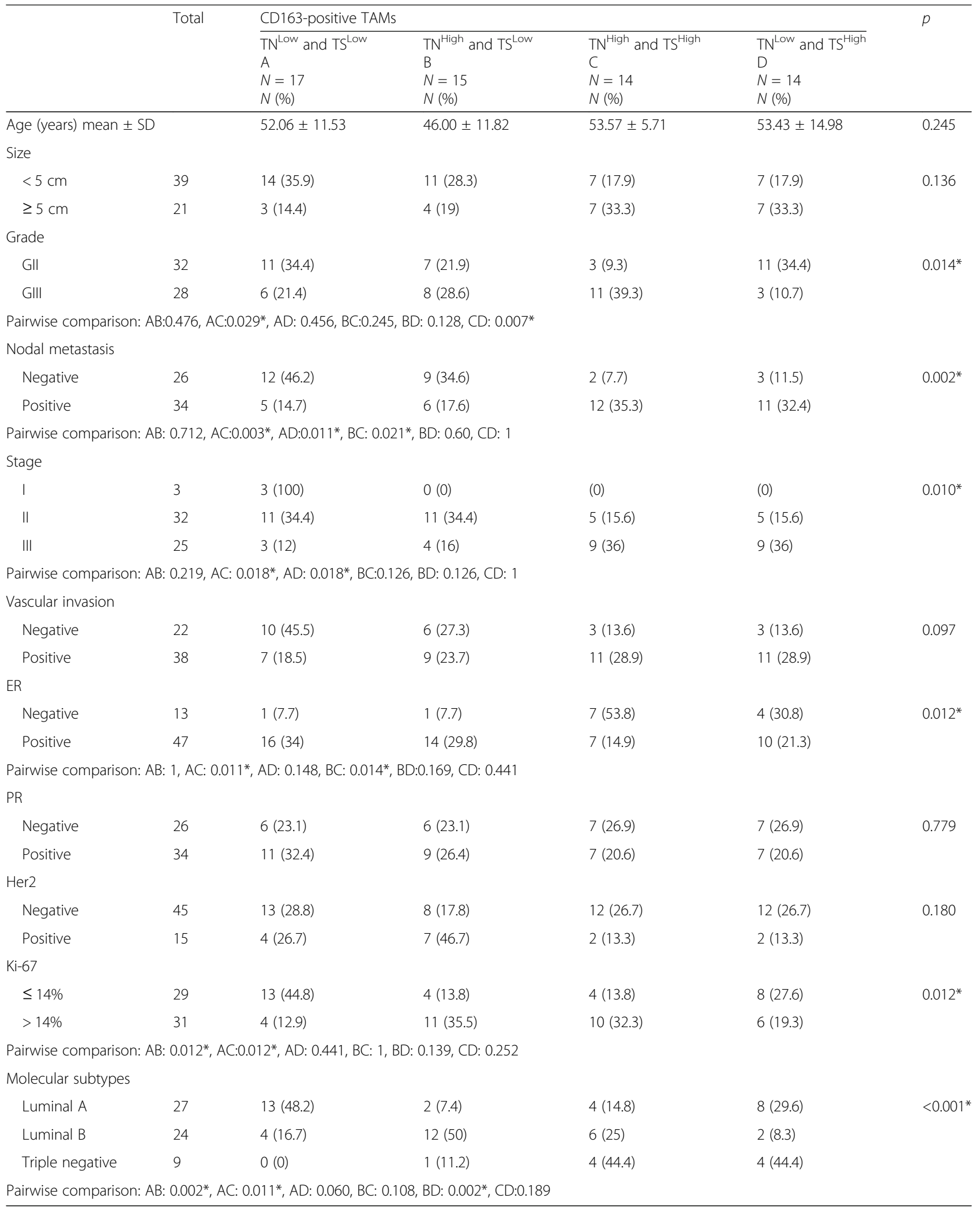

$E R$ estrogen receptor, $P R$ progesterone receptor, TAMs tumor-associated macrophage, $T N$ tumor nest, TS tumor stroma 


\section{Conclusion}

High density of CD163-positive stromal TAMs is strongly associated with positive vascular invasion, nodal metastasis, and molecular subtypes. Whereas high density of CD68-positive stromal TAMs is related to large tumor size and positive nodal metastasis. TAMs within tumor stroma and tumor nest have different levels of association with poor prognostic parameters. So, it is of great importance to consider the histologic localization of TAMs in addition to the degree of TAMs infiltration.

\section{Abbreviations \\ ANOVA: Analysis of variance; BC: Breast cancer; Cl: Confidence interval; DAB: Diaminobenzidine; ER: Estrogen receptor; HRP: Horseradish peroxidase; LN: Lymph node; OR: Odds ratio; PR: Progesterone receptor; TAMs: Tumor- associated macrophages; TME: Tumor microenvironment; TN: Tumor nest; TS: Tumor stroma; VEGF: Vascular endothelial growth factor; WHO: World Health Organization}

\section{Acknowledgements}

Not applicable

\section{Authors' contributions}

DMG participated in interpreting histopathologic and immunohistochemical stained slides, captured figures provided in this study, and was a major contributor in writing the manuscript. Also, participated in the design of the study and performed the statistical analysis. ShEM participated in interpreting histopathologic and immunohistochemical stained slides, captured figures provided in this study, and helped to draft the manuscript. Both authors read and approved the final manuscript.

\section{Funding}

Not applicable

\section{Availability of data and materials}

The datasets used and/or analyzed during the current study are available from the corresponding author on reasonable request.

\section{Ethics approval and consent to participate}

The protocol was approved by the Ethics Committee in the Faculty of Medicine, Tanta University [reference\# 33329] and before the initiation of any treatment, all patients signed an informed consent.

\section{Consent for publication}

Not applicable

\section{Competing interests}

The authors declare that they have no competing interests.

Received: 9 October 2019 Accepted: 8 January 2020

Published online: 27 January 2020

\section{References}

1. Bray F, Ferlay J, Soerjomataram I, Siegel RL, Torre LA, Jemal A. Global cancer statistics 2018: GLOBOCAN estimates of incidence and mortality worldwide for 36 cancers in 185 countries. CA Cancer J Clin. 2018;68(6):394-424.

2. Qiu SQ, Waaijer SJH, Zwager MC, de Vries EGE, van der Vegt B, Schröder CP. Tumor-associated macrophages in breast cancer: Innocent bystander or important player? Cancer Treat Rev. 2018;70:178-89.

3. Mittal K, Ebos J, Rini B. Angiogenesis and the Tumor Microenvironment: Vascular Endothelial Growth Factor and Beyond. Semin Oncol. 2014;41(2): 235-51

4. Kitamura T, Qian BZ, Pollard JW. Immune cell promotion of metastasis. Nat Rev Immunol. 2015;15(2):73-86.

5. Tang $X$. Tumor-associated macrophages as potential diagnostic and prognostic biomarkers in breast cancer. Cancer Lett. 2013;332(1):3-10.
6. Ambarus CA, Krausz S, van Eijk M, Hamann J, Radstake TR, Reedquist KA et al. Systematic validation of specific phenotypic markers for in vitro polarized human macrophages. J Immunol Methods. 2012;375(1):196-206.

7. Ruffell B, Coussens LM. Macrophages and therapeutic resistance in cancer. Cancer Cell. 2015;27(4):462-72

8. Mahmoud SM, Lee AH, Paish EC, Macmillan RD, Ellis IO, Green AR. Tumourinfiltrating macrophages and clinical outcome in breast cancer. J Clin Pathol. 2012;65(2):159-63.

9. Lakhani SE, Ellis IO, Schnitt SJ, Tan PH, van de Vijver MJ: WHO classification of tumors of the breast, vol. 4. Lyon, France: International Agency for Research on Cancer (IARC); 2012

10. Elston CW and Ellis IO: Assessment of histological grade. In Elston, CW and Ellis IO (eds), The breast. Vol. 13. Churchill Livingstone, Edinburgh; New York, 1998;356-84.

11. Giuliano AE, Edge SB, Hortobagyi GN. Eighth Edition of the AJCC Cancer Staging Manual: Breast Cancer. Ann Surg Oncol. 2018;25(7):1783-5.

12. Goldhirsch A, Wood WC, Coates AS, Gelber RD, Thürlimann B, Senn HJ, et al. Strategies for subtypes--dealing with the diversity of breast cancer: highlights of the St. Gallen International Expert Consensus on the Primary Therapy of Early Breast Cancer 2011. Ann Oncol 2011, 22(8):1736-1747.

13. Gwak JM, Jang MH, Kim DI, Seo AN, Park SY. Prognostic value of tumorassociated macrophages according to histologic locations and hormone receptor status in breast cancer. PLoS One. 2015;10(4):e0125728.

14. Pelekanou V, Villarroel-Espindola F, Schalper KA, Pusztai L, Rimm DL. CD68, CD163, and matrix metalloproteinase 9 (MMP-9) co-localization in breast tumor microenvironment predicts survival differently in ER-positive and -negative cancers. Breast Cancer Res. 2018;20(1):154.

15. Räihä MR, Puolakkainen PA. Tumor-associated macrophages (TAMs) as biomarkers for gastric cancer: A review. Chronic Dis Transl Med. 2018;4(3): 156-63.

16. Minami K, Hiwatashi K, Ueno S, Sakoda M, lino S, Okumura H, et al. Prognostic significance of CD68, CD163 and Folate receptor- $\beta$ positive macrophages in hepatocellular carcinoma. Exp Ther Med. 2018:15(5):4465-76.

17. Ch'ng ES, Tuan Sharif SE, Jaafar H. In human invasive breast ductal carcinoma, tumor stromal macrophages and tumor nest macrophages have distinct relationships with clinicopathological parameters and tumor angiogenesis. Virchows Arch. 2013;462(3):257-67.

18. Jeong H, Hwang I, Kang SH, Shin HC, Kwon SY. Tumor-Associated Macrophages as Potential Prognostic Biomarkers of Invasive Breast Cancer. J Breast Cancer. 2019;22(1):38-51.

19. Medrek C, Pontén F, Jirström K, Leandersson K. The presence of tumor associated macrophages in tumor stroma as a prognostic marker for breast cancer patients. BMC Cancer. 2012;12:306.

20. Ni C, Yang L, Xu Q, Yuan H, Wang W, Xia W, et al. CD68- and CD163positive tumor infiltrating macrophages in non-metastatic breast cancer: a retrospective study and meta-analysis. J Cancer. 2019;10(19):4463-72.

21. Sousa S, Brion R, Lintunen M, Kronqvist P, Sandholm J, Mönkkönen J, et al. Human breast cancer cells educate macrophages toward the M2 activation status. Breast Cancer Res. 2015;17(1):101.

22. Yang M, Li Z, Ren M, Li S, Zhang L, Zhang X, et al. Stromal Infiltration of Tumor-Associated Macrophages Conferring Poor Prognosis of Patients with Basal-Like Breast Carcinoma. J Cancer. 2018;9(13):2308-16.

23. Yuan ZY, Luo RZ, Peng RJ, Wang SS, Xue C. High infiltration of tumorassociated macrophages in triple-negative breast cancer is associated with a higher risk of distant metastasis. Onco Targets Ther. 2014;7:1475-80.

24. Stossi F, Madak-Erdoğan Z, Katzenellenbogen BS. Macrophage-elicited loss of estrogen receptor-a in breast cancer cells via involvement of MAPK and c-Jun at the ESR1 genomic locus. Oncogene. 2012;31(14):1825-34.

25. Yang J, Li X, Liu X, Liu Y. The role of tumor-associated macrophages in breast carcinoma invasion and metastasis. Int J Clin Exp Pathol. 2015;8(6): 6656-64.

26. Yang M, McKay D, Pollard JW, Lewis CE. Diverse Functions of Macrophages in Different Tumor Microenvironments. Cancer Res. 2018;78(19):5492-503.

27. Jackute J, Zemaitis M, Pranys D, Sitkauskiene B, Miliauskas S, Vaitkiene S, et al. Distribution of M1 and M2 macrophages in tumor islets and stroma in relation to prognosis of non-small cell lung cancer. BMC Immunol. 2018; 19(1):3

\section{Publisher's Note}

Springer Nature remains neutral with regard to jurisdictional claims in published maps and institutional affiliations. 